\title{
A study on alcohol dependence and it's determinants among male patients attending a tertiary care hospital, Kolkata
}

\author{
Sumit Mukherjee ${ }^{1}$, Sayanti Ghosh ${ }^{2}$ \\ ${ }^{1}$ Assistant Professor, Department of Psychiatry, IIMSAR, Haldia, ${ }^{2}$ Associate Professor, Department of Psychiatry, \\ N.R.S. Medical College, Kolkata, West Bengal, India
}

\section{A B S T R A C T}

Background: Alcohol intake is one of the major substance abuse and the problems of alcoholism is present throughout the world. There are some fundamental differences regarding socio-demographic profile, age of onset of drinking, and the nature of drinking etc. Aims and Objectives: This study was conducted to find out the association of several determinants like onset and duration of drinking, family history and role of biomarkers like Gamma-Glutamyl transferase (GGT), Mean corpuscular volume (MCV) with severity of alcohol dependence. Materials and Methods: It was a hospital based cross-sectional and observational study. Screening and assessment of alcohol dependence was done by using scales like AUDIT and Alcohol dependence scale. Semi-structured questioner was used for assessing sociodemographic data for the total sample of 100 patients. 65 cases were selected finally after screening by AUDIT scale. GGT and MCV estimation was done for the finally selected cases along with healthy controls. Descriptive statistical analysis was done for obtaining results. Results: Findings suggest that early onset of drinking that is 17.14 years is associated with severe level of alcohol dependence. Study shows that family history of alcohol use is a predictor of developing alcohol dependence. Biomarkers like GGT, $\mathrm{MCV}$ had shown significant elevation for cases of severe dependence. Conclusion: The burden of alcohol use is growing alarmingly in India and before it turns out to be a giant monster encroaching upon youths, we need to focus our attention in curbing this problem by diagnosing and treating alcohol dependent patients and using preventive measures, to not allow development of dependence at all.

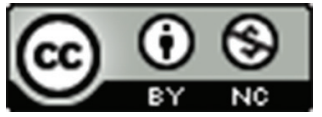

This work is licensed under a Creative Commons Attribution-NonCommercial 4.0 International License.

Key words: ADS; Alcohol dependence; AUDIT; Biomarkers; Onset of drinking

\section{INTRODUCTION}

Alcoholism is a major problem of the modern world and it is a leading preventable cause of death around the world. It is available in many forms with beer being the third most popular drink throughout the world next to water and tea. Nearly $5.1 \%$ of the global burden of disease is attributable to alcohol consumption, and it causes nearly 3.3 million deaths every year. ${ }^{1}$ The National Mental Health Survey conducted in 2015-2016 covered 12 states and found that alcohol use disorder is affecting $4.7 \%$ of the population in our country. ${ }^{2}$
Alcohol dependence is a cluster of behavioural, cognitive, and physiological phenomena that may develop after repeated alcohol use. ${ }^{3}$ Typically these phenomena include, a strong desire to consume alcohol, impaired control over its use, persistent despite harmful consequences, a higher priority given to drinking than to other activities and obligations, increased alcohol tolerance and a physical withdrawal reaction when alcohol is discontinued.

The use of alcohol is increasing with time not only in developed countries but also in the developing countries. The increased consumption of alcohol specially in developing countries like India is mainly due to changes in 
socio-cultural and economic environment. A large number of people specially from younger age group are consuming alcohol in a frequent manner. As many as $80 \%$ men and $60 \%$ women in developed countries drink at some time in their lives. The life time risk for development of alcohol use disorder in male is more than $20 \%$, with a risk of about $15 \%$ for alcohol abuse and 10\% for alcohol dependence. ${ }^{4}$

Alcohol consumption and subsequent development of dependence is associated with several factors like age of onset of drinking, family history of drinking, presence of any stressful life events, and socio-cultural back ground. A study conducted at National Institute of Mental Health and Neurosciences (NIMHANS), Bangalore, in 2005, it was found that $97 \%$ of alcohol dependents were Hindus, males, belonging to low socioeconomic class and had a strong family history of Alcohol dependence. ${ }^{5}$ Age of onset of drinking is associated with increased severity of alcoholism and later development of alcohol dependence.

Heavy substance use during adolescence is associated with substance use problems during adulthood. ${ }^{6}$ Research also suggests that adolescents who have an early onset of alcohol use are more likely to drink heavily and to develop alcohol problems than those who initiate alcohol use later. According to analyses of the National Longitudinal Alcohol Epidemiologic Study, a national survey of adults 18 years and older conducted in 1991-1992 $(n=42682$; response rate, 90\%), among persons who began drinking before age of 14 years, $45 \%$ developed Diagnostic and Statistical Manual of Mental Disorders, Fourth Edition, diagnosable dependence compared with $10 \%$ of persons who waited until they were 21 years or older. ${ }^{7}$ Early onset of drinking particularly in and around 13 years of age leads to a tendency of excessive drinking and intoxication in later part of life.

The relationship between early substance use and later substance abuse problems may simply reflect an individual's genetic predisposition for the development of substance abuse and dependence. ${ }^{8}$ Numerous studies have shown that individuals with a genetic predisposition for substance abuse (e.g., offspring of alcoholic parents) have an increased risk for early substance use, heavy substance use, and alcohol and drug problems.'

There are several biomarkers through which we can assess the severity of alcohol dependence as well as liver damage. Gamma-Glutamyl transferase (GGT), Mean corpuscular volume (MCV) are two important biomarkers among them. GGT is an enzyme and it is a protein biomarker where as MCV is a haematological parameter that is non-protein marker. GGT is one of the longest established biochemical tests for excessive alcohol consumption.
The MCV is elevated in approximately $50-60 \%$ of people who chronically ingest excessive alcohol quantities. With the advent of automated instruments that determine the MCV during routine blood counts, physicians frequently detect elevated MCV's in patients who are well nourished and who have no obvious disorders to explain this finding. A moderately increased MCV may be a clue to unsuspected alcoholism.

All these determinants should be studied in detail so that proper preventive measures could be taken to prevent alcohol dependence. There is very few study about the development of alcohol dependence in Indian context particularly in West Bengal. This study will help us to find out the exact correlation of these determinants and that knowledge will aid us in developing appropriate strategies to prevent this problem for the benefit of society.

\section{Aims and objectives}

The primary objective of this study was to identify the socio-demographic profile of alcohol dependent male patients and also to assess the severity of alcohol dependence in those male patients.

The secondary objective of this study was to find out the association between severity of alcohol dependence with the variables like age of onset of drinking, duration of drinking, family history of drinking etc as well as the role of biomarkers like GGT, MCV in determination of severity of alcohol dependence.

\section{MATERIALS AND METHODS}

The study was conducted at R.G. Kar Medical College Kolkata after obtaining approval from Ethics committee. It was a hospital based cross-sectional study. Patients attending General Medicine, Gastroenterology, and Orthopaedics OPD and IPD with complications secondary to alcohol use are usually referred to Psychiatry OPD after medical/ surgical intervention. Moreover some patients having alcohol use problems come directly to the Psychiatry OPD. Both these groups of patients with provisional diagnosis of disorders due to alcohol use according to ICD-10 and who fulfil the inclusion and exclusion criteria were included in the study. Purposive sampling was done for this study and data was collected for a period of 12 months. 100 cases have been selected who were having history of alcohol intake and among the cases 35 cases have been excluded from the study as they are not qualifying either in AUDIT scale or having some comorbid substance abuse excluding the tobacco. The male patients who belong to the age group of 20-50 years were included in the study. Also the patients who were suffering currently from any other major psychiatric disorders were excluded from this study. 
Finally 65 cases had been selected along with 65 healthy controls who were properly matched for age and sex for biochemical estimation. A pretested and predesigned semi-structured proforma containing questionnaire for socio-demographic profile was used. For assessment of socio-economic status of the family, Kuppuswamy scale (based on education and occupation of head of the family and family income) was used.

Alcohol dependence scale (ADS) was used to evaluate the severity of dependence. The ADS provides a quantitative measure of the severity of alcohol dependence consistent with the concept of the alcohol dependence syndrome. It measures severity in 4 quartiles like:

$1^{\text {st }}$ quartile: Low level of alcohol dependence

$2^{\text {nd }}$ quartile: Intermediate level of alcohol dependence

$3^{\text {rd }}$ quartile: Substantial level of alcohol dependence

$4^{\text {th }}$ quartile: Severe level of alcohol dependence

Biochemical estimation of GGT and MCV were done in laboratory from blood sample of patients and appropriately matched healthy controls. GGT and MCV estimation were done by bi-chromatic rate technique and automated analyser respectively.

GGT and MCV estimation were done by bi-chromatic rate technique and automated analyser respectively.

For statistical analysis data were entered into a Microsoft excel spreadsheet and then analysed by SPSS 20.0.1 and Graph Pad Prism version 5. Data had been summarized as mean and standard deviation for numerical variables and count and percentages for categorical variables. Two-sample t-tests for a difference in mean involved independent samples or unpaired samples was done. A Chi-squared test ( $\chi^{2}$ test) was any statistical hypothesis test wherein the sampling distribution of the test statistic is a Chi-squared distribution when the null hypothesis is true and was performed for data analysis. If the calculated P-value was below the threshold chosen for statistical significance (usually the 0.10 , the 0.05 , or 0.01 level), then the null hypothesis is rejected in favour of the alternative hypothesis. $\mathrm{P} \leq 0.05$ was considered for statistically significant.

\section{RESULTS}

In final study sample the mean age was 39.40 years and majority of the cases were from urban area. Among cases around $75 \%$ were married whereas around $15 \%$ were unmarried and few were separated. Association between marital status and severity of alcohol dependence is significant that is unmarried and separated individuals have severe dependence ( $\mathrm{P}$ value 0.0435). Family history of alcoholism is associated with severe level of dependence $(\mathrm{P}<0.00010)$ as described in (Table 1).

Majority of the cases belong from Hindu community and involved in unskilled work as shown in (Table 2).

Regarding educational status of the study population around $49 \%$ of the cases were having secondary level education, and only $9 \%$ of the cases had received education up to graduate level. A large number of cases belong from upper lower class in this study whereas there was no representation from upper class category.

The significant finding of this is study more than half of the cases had developed severe level of alcohol dependence and there was no case in the category of low level of alcohol dependence. A significant number of cases (41.5\%) were also in the category of substantial level of alcohol dependence. In this study also result suggests that early age of onset of drinking is significantly associated with severe level of alcohol dependence as shown in (Table 3). In this

\begin{tabular}{|c|c|c|c|c|}
\hline $\begin{array}{l}\text { Family } \\
\text { history }\end{array}$ & $2^{\text {nd }}$ quartile & $3^{\text {rd }}$ quartile & $4^{\text {th }}$ quartile & Total \\
\hline No & 3 & 17 & 1 & 21 \\
\hline Yes & 0 & 10 & 34 & 44 \\
\hline Total & 3 & 27 & 35 & 65 \\
\hline
\end{tabular}

\begin{tabular}{|c|c|c|c|c|}
\hline Occupation & $2^{\text {nd }}$ quartile & $3^{\text {rd }}$ quartile & $4^{\text {th }}$ quartile & Total \\
\hline Skilled & 3 & 5 & 6 & 14 \\
\hline Unskilled & 0 & 22 & 29 & 51 \\
\hline Total & 3 & 27 & 35 & 65 \\
\hline
\end{tabular}

\section{Table 3: Distribution of means of age of first onset of alcohol drinking with ADS level}

\begin{tabular}{|c|c|c|c|c|c|c|c|}
\hline ADS level & Number & Mean & SD & Minimum & Maximum & Median & P-value \\
\hline $2^{\text {nd }}$ quartile & 3 & 26.6667 & 1.1547 & 26.0000 & 28.0000 & 26.0000 & $<0.0001$ \\
\hline $3^{\text {rd }}$ quartile & 27 & 23.4444 & 1.3681 & 20.0000 & 26.0000 & 24.0000 & \\
\hline $4^{\text {th }}$ quartile & 35 & 17.1429 & 1.7002 & 15.0000 & 24.0000 & 17.0000 & \\
\hline
\end{tabular}

ADS: Alcohol dependence scale 
study the patients who are having level 4 dependence that is severe level dependence the mean age of onset is much lower that is 17.14 years where as patients with intermediate level of dependence having mean onset at age 26.66 years.

Those patients who were having history of severe level dependence had history of more than 20 months of mean duration of continuous drinking as shown in (Table 4). Statistically significant result has been noted for the duration of continuous drinking and severity level of dependence.

In previous studies for GGT it has been noticed that raised GGT level may be associated with more severe liver damage rather more severe level of alcohol dependence. Mean GGT value for severe level of alcohol dependence was 121.37 $\mathrm{U} / \mathrm{L}$ whereas for intermediate level of dependence the mean value is much less $50.66 \mathrm{U} / \mathrm{L}$ and these difference in mean is statistically significant whereas the reference range for GGT for male is (11-51) U/L. It can be interpreted that people with more severe level of alcohol dependence will have higher value of GGT as shown (Table 5). Result suggests that mean GGT value between case and healthy control is statistically significant $(\mathrm{P}<0.0001)$ and cases have higher value of GGT described in (Table 6).

The mean volume of the red blood cell (MCV) has been recognized for many years as increasing with excessive alcohol consumption. The mean MCV value for patients who were suffering from alcohol dependence were $96.47 \mathrm{fL}$, and for control group was $88.16 \mathrm{fL}$ which was statistically significant $(\mathrm{P}<0.0001)$. The reference range for MCV for adults is (82-96) fL. The change in mean difference of $\mathrm{MCV}$ in case and control group is statistically significant as described in (Table 7). Analysis of MCV with severity of alcohol dependence also suggests that more severe level of dependence alter MCV significantly as shown in (Table 8).

Table 4: Distribution of means of continuous drinking in month with ADS level

\begin{tabular}{cccccccc}
\hline ADS level & Number & Mean & SD & Minimum & Maximum & Median & P-value \\
\hline $2^{\text {nd }}$ quartile & 3 & 5.3333 & 0.5774 & 5.0000 & 6.0000 & 5.0000 & $<0.0001$ \\
$3^{\text {rd }}$ quartile & 27 & 11.5556 & 1.3107 & 9.0000 & 14.0000 & 12.0000 & 20.0000 \\
$4^{\text {th }}$ quartile & 35 & 20.7143 & 2.3461 & 17.0000 & 26.0000 & \\
\hline
\end{tabular}

ADS: Alcohol dependence scale

Table 5: Distribution of means of GGT with ADS level

\begin{tabular}{lccccccc}
\hline ADS level & Number & Mean & SD & Minimum & Maximum & Median & P-value \\
\hline $2^{\text {nd }}$ quartile & 3 & 50.6667 & 11.9304 & 41.0000 & 64.0000 & 47.0000 & $<0.0001$ \\
$3^{\text {rd }}$ quartile & 27 & 82.4815 & 9.4843 & 61.0000 & 97.0000 & 85.0000 & 106.0000 \\
$4^{\text {th }}$ quartile & 35 & 121.3714 & 33.8579 & 87.0000 & 212.0000 & \\
\hline
\end{tabular}

GGT: Gamma-Glutamyl transferase, ADS: Alcohol dependence scale

Table 6: Distribution of means of GGT in two groups

\begin{tabular}{lccccccc} 
GGT group & Number & Mean & SD & Minimum & Maximum & Median & P-value \\
\hline Case & 65 & 101.9538 & 33.7558 & 41.0000 & 212.0000 & 93.0000 & $<0.0001$ \\
Control & 65 & 21.5538 & 9.6226 & 9.0000 & 49.0000 & 18.0000 & \\
\hline
\end{tabular}

GGT: Gamma-Glutamyl transferase

Table 7: Distribution of means of MCV in two groups

\begin{tabular}{lccccccc}
\hline MCV group & Number & Mean & SD & Minimum & Maximum & Median & P-value \\
\hline Case & 65 & 96.4769 & 2.4502 & 90.0000 & 100.0000 & 97.0000 & $<0.0001$ \\
Control & 65 & 88.1692 & 3.8102 & 80.0000 & 97.0000 & 88.0000 & \\
\hline
\end{tabular}

MCV: Mean corpuscular volume

Table 8: Distribution of means of MCV with ADS level

\begin{tabular}{lccccccc}
\hline ADS level & Number & Mean & SD & Minimum & Maximum & Median & P-value \\
\hline $2^{\text {nd }}$ quartile & 3 & 93.0000 & 3.6056 & 90.0000 & 97.0000 & 92.0000 & 0.0158 \\
$3^{\text {rd }}$ quartile & 27 & 96.1852 & 1.9223 & 92.0000 & 100.0000 & 97.0000 & 97.0000 \\
$4^{\text {th }}$ quartile & 35 & 97.0000 & 2.5088 & 91.0000 & 100.0000 & \\
\hline
\end{tabular}

MCV: Mean corpuscular volume, ADS: Alcohol dependence scale 


\section{DISCUSSION}

This study deals with the role of different determinants including socio-demographic, biochemical factors in development of alcohol dependence in males who are attending a tertiary care hospital in a metropolitan city.

The significant finding of this study is more than half of the cases had developed severe level of alcohol dependence and there was no case in the category of low level of alcohol dependence. A significant number of cases (41.5\%) were also in the category of substantial level of alcohol dependence.

It can be opined from this finding that majority of cases who had developed intermediate and low level of alcohol dependence may not have attended the out-patient departments may be because of they had not developed significant impairment in daily life or deterioration in general health condition.

Regarding family history of alcoholism it has been seen in previous studies like Dhupdale et al., that an alcoholic father was a great risk factor for the development of alcohol dependence in children. ${ }^{10}$ Goodwin (1984) reported that familial alcoholism is associated with early onset and severe course of dependence. ${ }^{11}$ Meena et al., in their study conducted in Rohtak found that $23.16 \%$ of alcohol users had an alcoholic father and $7.5 \%$ had alcoholic grandfather. ${ }^{12}$ According to our study a significant number of patients with severe level of alcohol dependence have reported family history of alcohol consumption. It can be said that family history itself is a strong risk factor for development of alcohol dependence. Regarding occupational and socio-economic status previous studies in India like Tiwari et al., (2003) have reported that people from low socio-economic class and males are most susceptible for harmful alcohol abuse and development of dependence. ${ }^{13}$ In developed country like Australia Queensland Government report suggests that a meaningful occupation is required for good physical and mental health. ${ }^{14}$ Poverty, unemployment, dissatisfaction in job may all lead to development of alcohol dependence. In this study findings suggest that patients who were unskilled worker had developed more severe alcohol dependence in significant manner. Though previous studies have shown association between socio-economic status with alcohol dependence for this study the results are nonsignificant from statistical point of view and it may be due to small sample size. It can be concluded that to establish the effects of education and socio-economic status large community based studies are required.

In this study majority of the cases were from Hindu community and few cases were from Muslim community.
Among the cases from Muslim community it has been seen that comparatively lesser number patients were suffering from severe alcohol dependence. In this study we could not find any statistical significance with severity of alcohol dependence and religion but it can be said that number alcohol users are much less in Muslim community. According to a study conducted at NIMHANS, Bangalore, in 2005 , it was found that $97 \%$ of alcohol dependents were Hindus, males and belongs from lower socio-economic class. $^{5}$

Regarding drinking pattern the most important thing is the age of first onset for alcohol drinking. It has also been seen that if the patients are having a history of long duration of continuous drinking has possibility to develop severe level of alcohol dependence in future. Reboussin et al., (2006) found in their study that young adulthood (ages 18-20 years) was significantly associated with regular problem drinking and suggested that the underage problem drinking was most strongly characterized by heavy drinking behaviours. ${ }^{15}$ Pitkanen et al., (2005) described the relationship between age of onset of drinking was a significant risk factor for high consumption of alcohol and problem drinking in adulthood. ${ }^{16}$

In this study results clearly suggest that early age of onset of drinking is significantly associated with severe level of alcohol dependence. The patients who are having level 4 dependence that is severe level dependence the mean age of onset is much lower that is 17.14 years where as patients with intermediate level of dependence having mean onset at age 26.66 years. Those patients who were having history of severe level dependence had history of more than 20 months of mean duration of continuous drinking. Statistically significant result has been noted for the duration of continuous drinking and development of severe level of dependence.

Biomarkers are measurable indicator of the severity or presence of some disease state. In case of alcohol dependence there are several biomarkers like MCV, GGT, Carbohydrate deficit transferrin etc. GGT is one of the longest established biochemical tests for excessive alcohol consumption. ${ }^{17}$ In this study during comparison of mean GGT value for cases and healthy controls, the mean value of GGT for cases are significantly higher than healthy controls. In previous studies for GGT it has been noticed that raised GGT level may be associated with more severe liver damage rather more severe level of alcohol dependence. ${ }^{18}$ Mean GGT value for severe level of alcohol dependence was $121.37 \mathrm{U} / \mathrm{L}$ whereas for intermediate level of dependence the mean value is much less $50.66 \mathrm{U} / \mathrm{L}$ and these difference in mean is statistically significant whereas the reference range for GGT for male is (11-51) U/L. It 
can be interpreted that people with more severe level of alcohol dependence will have higher value of GGT.

The mean volume of the red blood cell (MCV) has been recognized for many years as increasing with excessive alcohol consumption. In alcohol excess, the majority of cases of macrocytosis occur in the presence of normal folate levels and without anaemia, and do not respond to folate treatment. ${ }^{19}$ The cause of macrocytosis is complex. Similar findings with statistical significance is noted for $\mathrm{MCV}$ while computing with severity of alcohol dependence. The mean MCV value for patients who were suffering from alcohol dependence were $96.47 \mathrm{fL}$, and for control group was $88.16 \mathrm{fL}$. The reference range for MCV for adults is (82-96) fL. The change in mean difference of MCV in case and control group is statistically significant. Analysis of MCV with severity of alcohol dependence also suggests that more severe level of dependence alter MCV significantly.

\section{Limitations of the study}

The sample size was very small and previous studies about alcohol dependence involved large number of sample from hospital/clinic as well as from community. The patients who are attending for treatment in this Government hospital usually belong to low socioeconomic status and because of that the results cannot be generalised for entire community. The patients who were having comparatively lesser degree of alcohol dependence may not have been referred to or attended the Psychiatry out patient department and there is a possibility of selection bias. All subjects in this study were males and findings may differ with inclusion of female patients. Lastly, apart from chronic alcoholism there are multiple causes of raised MCV like deficiency in Vitamin B12, Folate, Myelodysplastic syndrome and all these aetiologies could not be excluded.

\section{CONCLUSION}

It is evident that the role of the family and school is pivotal in eliminating this habit of dependence of alcohol as it will prevent the alcohol intake in early period of life and the chance of development of alcohol dependence. School based programmes can be initiated to generate awareness regarding the potential health hazards. Educational material regarding alcohol abuse and their harmful effects can be imparted to teachers and later introduced in school curriculum. More research in community in large scale is required to identify the exact role of sociocultural and economic aspects in development of harmful alcohol use. Further robust research is necessary regarding biomarkers of alcohol dependence and that will lead to better understanding of pathophysiology of alcohol related harms.
Unless planned policy changes are designed and vigorously implemented these problems are likely to produce excessive burden in the resource of developing countries.

Alcohol menace is growing alarmingly in India and before it turns out to be a giant monster encroaching upon our youths, we need to focus our attention in curbing this problem not only by diagnosing and treating alcohol dependent patients by proper interventions but also to tackle the promotion of alcohol so as to not allow development of dependence at all.

\section{ACKNOWLEDGMENT}

The authors are grateful to the staffs and faculty members of Psychiatric, Biochemistry and Pathology department of R.G.Kar Medical College and Hospital for their help and support for conducting the study.

\section{REFERENCES}

1. Rehm J, Gmel GE Sr., Gmel G, Hasan OSM, Imtiaz S, Popova $S$, et al. The relationship between different dimensions of alcohol use and the burden of disease-an update. Addiction. 2017;112(6):968-1001.

https://doi.org/10.1111/add.13757

2. Gururaj G, Varghese M, Benegal V, Rao GN, Pathak K, Singh LK, et al. National Mental Health Survey of India, 2015-16: Prevalence, Patterns and Outcomes. Bengaluru: NIMHANS; 2016.

3. World Health Organization. The ICD-10 Classification of Mental and Behavioural Disorders: Diagnostic Criteria for Research. Geneva: World Health Organization; 1993.

4. World Health Organization. Management of Substance Abuse Unit. Global Status Report on Alcohol and Health. Luxembourg: WHO Press; 2014.

5. Gururaj G, Murthy P, Girish N and Benegal V. Alcohol Related Harm: Implications of Public Health and Policy in India, Publication No. 73. Bangalore: NIMHANS; 2011.

6. Brook DW, Brook JS, Zhang C, Cohen P and Whiteman M. Drug use and the risk of major depressive disorder, alcohol dependence and substance use disorders. Arch Gen Psychiatry. 2002;59(11):1039-1044.

https://doi.org/10.1001/archpsyc.59.11.1039

7. Grant BF and Dawson DA. Age at onset of alcohol use and its association with DSM-IV alcohol abuse and dependence: Results from the national longitudinal alcohol epidemiologic survey. J Subst Abuse. 1997;9:103-110. https://doi.org/10.1016/S0899-3289(97)90009-2

8. Heath AC, Madden PA, Bucholz KK, Dinwiddie $\mathrm{SH}$, Slutske WS, Bierut LJ, et al. Genetic and environmental risks of dependence on alcohol, tobacco, and other drugs. In: Plomin R, DeFries JC, Craig IW, McGuffin P, editors. Behavioral Genetics in the Postgenomic Era. Washington, DC: American Psychological Association; 2003. p. 309-334. https://doi.org/10.1037/10480-017

9. Cloninger CR, Bohman $\mathrm{M}$ and Sigvardsson S. Inheritance of alcohol abuse. Cross-fostering analysis of adopted men. Arch Gen Psychiatry. 1981;38(8):861-868.

https://doi.org/10.1001/archpsyc.1981.01780330019001 
10. Dhupdale NY, Motghare DD, Prasad YD and Feereira AM. Prevalence and pattern of alcohol use in Rural Goa. Indian J Community Med. 2006;31(2):104-105.

11. Goodwin DA. Studies of familial alcoholism: A review. J Clin Psychiatry. 1984;45(2):14-17.

12. Meena, Khanna P, Vohra AK and Rajput R. Prevalence and pattern of alcohol and substance abuse in urban areas of Rohtak city. Indian J Psychiatry. 2002;44(4):348-352.

13. Tiwari SC, Kumar A, Tripathi RK, Kumar R and Srivastava G. Profile of neuropsychiatric morbidity amongst urban and rural elderly (preliminary observations). Indian J Geriatr Ment Health. 2010;2:11-20.

14. Queensland Mental Health, Alcohol and Other Drugs Strategic Plan 2018-2023: Queensland Mental Health Commission Publication; 2018.

15. Reboussin BA, Song EY, Shrestha A, Lohman KK and Wolfson MA. A latent class analysis of underage problem drinking: Evidence from a community sample of $16-20$ years old. Drug Alcohol Depend. 2006;83(3):199-209. https://doi.org/10.1016/j.drugalcdep.2005.11.013

16. Pitkanen $T$, Lyyra $A L$ and Pulkkinen $L$. Age of onset of drinking and the use of alcohol in adulthood: A follow-up study from age 8-42 for females and males. Addiction. 2005;100(5):652-661. https://doi.org/10.1111/j.1360-0443.2005.01053.x

17. Rosalki SB, Rau D, Lehmann D and Prentice M. Determination of serum gamma-glutamyl transpeptidase activity and its clinical applications. Ann Clin Biochem. 1970;7:143-147. https://doi.org/10.1177/000456327000700601

18. Sillanaukee P, Massot N, Jousilahti P, Vartiainen E, Sundvall J, Olsson $U$, et al. Dose-response of laboratory markers to alcohol consumption in a general population. Am J Epidemiol. 2000;152(8):747-751.

https://doi.org/10.1093/aje/152.8.747

19. Wu A, Chanarin I and Levi AJ. Macrocytosis of chronic alcoholism. Lancet. 1974;1(7862):829-830. https://doi.org/10.1016/S0140-6736(74)90482-6

Authors Contribution:

SM- Concept, data collection, reviewed the literature, statistical analysis and interpretation of the results, preparation of manuscript; SG- Concept and design of the study, coordination, revision of manuscript

Work attributed to:

R.G.Kar Medical College and Hospital, Kolkata

Orcid ID:

Dr. Sumit Mukherjee- (1) https://orcid.org/0000-0003-4708-7134

Dr. Sayanti Ghosh- (iD https://orcid.org/0000-0002-7227-556X

Source of Support: None, Conflict of Interest: None. 